\title{
A Weighted Version of the Mishou Theorem
}

\author{
Antanas Laurinčikas ${ }^{a}$, Darius Šiaučiūnas ${ }^{b}$ and \\ Gediminas Vadeikis ${ }^{a}$ \\ ${ }^{a}$ Institute of Mathematics, Faculty of Mathematics and Informatics, Vilnius \\ University \\ Naugarduko g. 24, LT-03225 Vilnius, Lithuania \\ ${ }^{b}$ Institute of Regional Development, Šiauliai University \\ P. Višinskio g. 25, LT-76351 Šiauliai, Lithuania \\ E-mail(corresp.): darius.siauciunas@su.It \\ E-mail: antanas.laurincikas@mif .vu.1t \\ E-mail: gediminas.vadeikis@mif.vu.lt
}

Received April 9, 2020; revised September 27, 2020; accepted September 27, 2020

\begin{abstract}
In 2007, H. Mishou obtained a joint universality theorem for the Riemann and Hurwitz zeta-functions $\zeta(s)$ and $\zeta(s, \alpha)$ with transcendental parameter $\alpha$ on the approximation of a pair of analytic functions by shifts $(\zeta(s+i \tau), \zeta(s+i \tau, \alpha))$, $\tau \in \mathbb{R}$. In the paper, the Mishou theorem is generalized for the set of above shifts having a weighted positive lower density. Also, the case of a positive density is considered.
\end{abstract}

Keywords: Hurwitz zeta-function, Mishou theorem, Riemann zeta-function, universality.

AMS Subject Classification: 11M06; 11M41.

\section{Introduction}

The Riemann zeta-function $\zeta(s), s=\sigma+i t$, and the Hurwitz zeta-function $\zeta(s, \alpha)$ with parameter $0<\alpha \leqslant 1$ are defined, for $\sigma>1$, by the Dirichlet series

$$
\zeta(s)=\sum_{m=1}^{\infty} \frac{1}{m^{s}} \quad \text { and } \quad \zeta(s, \alpha)=\sum_{m=0}^{\infty} \frac{1}{(m+\alpha)^{s}},
$$

and have analytic continuation to the whole complex plane, except for a simple pole at the point $s=1$ with residue 1 . The functions $\zeta(s)$ and $\zeta(s, \alpha)$ play an important role not only in analytic number theory but in mathematics in

Copyright (C) 2021 The Author(s). Published by Vilnius Gediminas Technical University

This is an Open Access article distributed under the terms of the Creative Commons Attribution License (http://creativecommons.org/licenses/by/4.0/), which permits unrestricted use, distribution, and reproduction in any medium, provided the original author and source are credited. 
general. The definitions of $\zeta(s)$ and $\zeta(s, \alpha)$ are similar, however, their analytic properties are quite different. For example, since the function $\zeta(s)$, for $\sigma>1$, has the Euler product over primes

$$
\zeta(s)=\prod_{p}\left(1-\frac{1}{p^{s}}\right)^{-1}
$$

$\zeta(s) \neq 0$ in the half-plane $\sigma>1$, while the function $\zeta(s, \alpha)$ has zeros in that half plane if $\alpha \neq 1$ or $1 / 2$. On the other hand, the functions $\zeta(s)$ and $\zeta(s, \alpha)$ have a common feature, they are universal in the sense that their shifts $\zeta(s+i \tau)$ and $\zeta(s+i \tau, \alpha)$ approximate wide classes of analytic functions. We recall that universality of the function $\zeta(s)$ was discovered by S.M. Voronin in [31]. For a statement of the Voronin theorem, it is convenient to use the following notation. For $D=\{s \in \mathbb{C}: 1 / 2<\sigma<1\}$, denote by $\mathcal{K}$ the class of compact subsets of the strip $D$ with connected complements, by $H(K)$ with $K \in \mathcal{K}$ the class of continuous functions on $K$ that are analytic in the interior of $K$, and by $H_{0}(K)$ the subclass of $H(K)$ of non-vanishing functions on $K$. Then the modern version of the Voronin theorem, see, for example, [1,6,13,30] asserts that, for every $K \in \mathcal{K}, f(s) \in H_{0}(K)$, and $\varepsilon>0$,

$$
\liminf _{T \rightarrow \infty} \frac{1}{T} \operatorname{meas}\left\{\tau \in[0, T]: \sup _{s \in K}|\zeta(s+i \tau)-f(s)|<\varepsilon\right\}>0 .
$$

The latter inequality shows that there are infinitely many shifts $\zeta(s+i \tau)$ approximating a given function $f(s) \in H_{0}(K)$. Here meas $A$ denotes the Lebesgue measure of a measurable set $A \subset \mathbb{R}$.

Universality of the Hurwitz zeta-function is a more complicated problem. At the moment, the following result is known. Suppose that $\alpha$ is a transcendental or rational $\neq 1,1 / 2$. Then, for every $K \in \mathcal{K}, f(s) \in H(K)$, and $\varepsilon>0$,

$$
\liminf _{T \rightarrow \infty} \frac{1}{T} \text { meas }\left\{\tau \in[0, T]: \sup _{s \in K}|\zeta(s+i \tau, \alpha)-f(s)|<\varepsilon\right\}>0 .
$$

The case of rational $\alpha$ was obtained by Voronin [32] and B. Bagchi [1], while the case of transcendental $\alpha$ was treated by S.M. Gonek [6], and, by a different method, in [23]. In [14], the transcendence of $\alpha$ was replaced by a weaker condition on the linear independence of the set $L(\alpha)=\left\{\log (m+\alpha): m \in \mathbb{N}_{0}=\right.$ $\mathbb{N} \cup\{0\}\}$ over the field of rational numbers $\mathbb{Q}$.

H. Mishou in [29] began to study a joint approximation property of the functions $\zeta(s)$ and $\zeta(s, \alpha)$. More precisely, he proved that if $\alpha$ is transcendental, then, for every $K_{1}, K_{2} \in \mathcal{K}, f_{1}(s) \in H_{0}\left(K_{1}\right), f_{2} \in H\left(K_{2}\right)$ and $\varepsilon>0$,

$$
\begin{array}{r}
\liminf _{T \rightarrow \infty} \frac{1}{T} \operatorname{meas}\left\{\tau \in[0, T]: \sup _{s \in K_{1}}|\zeta(s+i \tau)-f(s)|<\varepsilon,\right. \\
\left.\sup _{s \in K_{2}}|\zeta(s+i \tau, \alpha)-f(s)|<\varepsilon\right\}>0 .
\end{array}
$$

The Mishou theorem is the first so-called mixed joint universality theorem because the function $\zeta(s)$ has Euler's product over primes, while the function 
$\zeta(s, \alpha)$ with transcendental $\alpha$ has no such a product. Mixed joint universality theorems were studied in $[2,5,7,8,9,10,11,15,16,17,18,19,20,21,22,24]$.

The aim of this paper, is a joint weighted universality theorem for the functions $\zeta(s)$ and $\zeta(s, \alpha)$. The weighted universality of zeta-functions was began to study in [12]. In weighted universality theorems, the positivity of a lower density of the shifts approximating a given analytic function is replaced by the positivity of that weighted analogue. Let $w(\tau)$ be positive function for $\tau \geqslant T_{0}>0$ such that

$$
\lim _{T \rightarrow \infty} W(T, w)=\infty, \quad W(T, w)=\int_{T_{0}}^{T} w(\tau) \mathrm{d} \tau,
$$

and, for every interval $[a, b] \subset\left[T_{0}, \infty\right)$, the variation $\mathrm{V}_{a}^{b} w$ satisfies the inequality $\mathrm{V}_{a}^{b} w \leqslant c w(a)$ with certain $c>0$. Moreover, let $I(A)$ denote the indicator function of the set $A$. Under the above hypotheses on the weight function $w$, it was obtained in [12] that, for every $K \in \mathcal{K}, f(s) \in H_{0}(K)$, and $\varepsilon>0$,

$$
\liminf _{T \rightarrow \infty} \frac{1}{W(T, w)} \int_{T_{0}}^{T} w(\tau) I\left(\left\{\tau \in\left[T_{0}, T\right]: \sup _{s \in K}|\zeta(s+i \tau)-f(s)|<\varepsilon\right\}\right) \mathrm{d} \tau>0 .
$$

A weighted discrete universality for $\zeta(s)$ was proved in [25]. Weighted universality theorems for periodic zeta-functions were obtained in [26,27].

A weighted universality theorem for the Hurwitz zeta-function was proved in [3]. Denote by $W$ the above class of weight functions.

Theorem 1. Suppose that $\alpha$ is transcendental and $w \in W$. Let $K \in \mathcal{K}$ and $f(s) \in H(K)$. Then, for every $\varepsilon>0$,

$$
\liminf _{T \rightarrow \infty} \frac{1}{W(T, w)} \int_{T_{0}}^{T} w(\tau) I\left(\left\{\tau \in\left[T_{0}, T\right]: \sup _{s \in K}|\zeta(s+i \tau, \alpha)-f(s)|<\varepsilon\right\}\right) \mathrm{d} \tau>0 .
$$

The main result of this paper is the following weighted theorem.

Theorem 2. Suppose that $\alpha$ is transcendental and $w \in W$. Let $K_{1}, K_{2} \in \mathcal{K}$ and $f(s) \in H_{0}\left(K_{1}\right), f_{2}(s) \in H\left(K_{2}\right)$. Then, for every $\varepsilon>0$,

$$
\begin{array}{r}
\liminf _{T \rightarrow \infty} \frac{1}{W(T, w)} \int_{T_{0}}^{T} w(\tau) I\left(\left\{\tau \in\left[T_{0}, T\right]: \sup _{s \in K_{1}}|\zeta(s+i \tau)-f(s)|<\varepsilon,\right.\right. \\
\left.\left.\sup _{s \in K_{2}}|\zeta(s+i \tau, \alpha)-f(s)|<\varepsilon\right\}\right) \mathrm{d} \tau>0 .
\end{array}
$$

Moreover, the limit

$$
\begin{array}{r}
\lim _{T \rightarrow \infty} \frac{1}{W(T, w)} \int_{T_{0}}^{T} w(\tau) I\left(\left\{\tau \in\left[T_{0}, T\right]: \sup _{s \in K_{1}}|\zeta(s+i \tau)-f(s)|<\varepsilon,\right.\right. \\
\left.\left.\sup _{s \in K_{2}}|\zeta(s+i \tau, \alpha)-f(s)|<\varepsilon\right\}\right) \mathrm{d} \tau>0
\end{array}
$$

exists for all but at most countably many $\varepsilon>0$.

If $w(\tau)=1$, then the first assertion of Theorem 2 reduces to the Mishou theorem [29]. For example, we may take $w(\tau)=1 / \tau$ and $\alpha=1 /$ e.

For the proof of Theorem 2, we will use the probabilistic approach based on weak convergence of probability measures in the space of analytic functions. 


\section{A weighted limit theorem on the product of two tori}

In what follows, we denote by $\mathcal{B}(\mathbb{X})$ the Borel $\sigma$-field of the space $\mathbb{X}$, by $\mathbb{P}$ the set of all prime numbers, and $\mathbb{N}_{0}=\mathbb{N} \cup\{0\}$.

Let $\gamma=\{s \in \mathbb{C}:|s|=1\}$. Define two tori $\Omega_{1}=\prod_{p \in \mathbb{P}} \gamma_{p}$ and $\Omega_{2}=$ $\prod_{m \in \mathbb{N}_{0}} \gamma_{m}$, where $\gamma_{p}=\gamma$ for all $p \in \mathbb{P}$ and $\gamma_{m}=\gamma$ for all $m \in \mathbb{N}_{0}$. With product topology and pointwise multiplication, the infinite-dimensional tori $\Omega_{1}$ and $\Omega_{2}$ are compact topological Abelian groups. Therefore, $\Omega=\Omega_{1} \times$ $\Omega_{2}$ is again a compact topological Abelian group. Hence, on $(\Omega, \mathcal{B}(\Omega))$, the probability Haar measure $m_{H}$ can be defined, and we obtain the probability space $\left(\Omega, \mathcal{B}(\Omega), m_{H}\right)$. Denote by $\omega_{1}(p)$ the $p$ th component of an element $\omega_{1} \in$ $\Omega_{1}, p \in \mathbb{P}$, and by $\omega_{2}(m)$ the $m$ th component of an element $\omega_{2} \in \Omega_{2}, m \in \mathbb{N}_{0}$. The elements of $\Omega$ are of the form $\omega=\left(\omega_{1}, \omega_{2}\right)$.

In this section, we will consider the weak convergence for

$$
\begin{array}{r}
Q_{T, w}(A)=\frac{1}{W(T, w)} \int_{T_{0}}^{T} w(\tau) I\left(\left\{\tau \in\left[T_{0}, T\right]:\left(\left(p^{-i \tau}: p \in \mathbb{P}\right),\right.\right.\right. \\
\left.\left.\left.\left((m+\alpha)^{-i \tau}: m \in \mathbb{N}_{0}\right)\right) \in A\right\}\right) \mathrm{d} \tau, \quad A \in \mathcal{B}(\Omega) .
\end{array}
$$

Theorem 3. Suppose that $\alpha$ is transcendental and $w \in W$. Then $Q_{T, w}$ converges weakly to the Haar measure $m_{H}$ as $T \rightarrow \infty$.

Proof. The characters of the group $\Omega$ are of the form

$$
\prod_{p \in \mathbb{P}}^{\prime} \omega_{1}^{k_{p}}(p) \prod_{m \in \mathbb{N}_{0}}^{\prime} \omega_{2}^{l_{m}}(m)
$$

where the sign "' " means that only a finite number of integers $k_{p}$ and $l_{m}$ are distinct from zero. Therefore, the Fourier transform $g_{T, w}(\underline{k}, \underline{l}), \underline{k}=\left(k_{p}: k_{p} \in\right.$ $\mathbb{Z}, p \in \mathbb{P}), \underline{l}=\left(k_{p}: l_{m} \in \mathbb{Z}, m \in \mathbb{N}_{0}\right)$, of $Q_{T, w}$ is defined by

$$
g_{T, w}(\underline{k}, \underline{l})=\int_{\Omega} \prod_{p \in \mathbb{P}}^{\prime} \omega_{1}^{k_{p}}(p) \prod_{m \in \mathbb{N}_{0}}^{\prime} \omega_{2}^{l_{m}}(m) \mathrm{d} Q_{T, w}
$$

Therefore, by the definition of $Q_{T, w}$,

$$
\begin{aligned}
& g_{T, w}(\underline{k}, \underline{l})=\frac{1}{W(T, w)} \int_{T_{0}}^{T} w(\tau) \prod_{p \in \mathbb{P}}^{\prime} p^{-i k_{p} \tau} \prod_{m \in \mathbb{N}_{0}}^{\prime}(m+\alpha)^{-i l_{m} \tau} \mathrm{d} \tau \\
& =\frac{1}{W(T, w)} \int_{T_{0}}^{T} w(\tau) \exp \left\{-i \tau\left(\sum_{p \in \mathbb{P}}^{\prime} k_{p} \log p+\sum_{m \in \mathbb{N}_{0}}^{\prime} l_{m} \log (m+\alpha)\right)\right\} \mathrm{d} \tau .
\end{aligned}
$$

Clearly,

$$
g_{T, w}(\underline{0}, \underline{0})=\frac{1}{W(T, w)} \int_{T_{0}}^{T} w(\tau) \mathrm{d} \tau=1 .
$$


Suppose that $(\underline{k}, \underline{l}) \neq(\underline{0}, \underline{0})$. Then

$$
A(\underline{k}, \underline{l}) \stackrel{\text { def }}{=} \sum_{p \in \mathbb{P}}^{\prime} k_{p} \log p+\sum_{m \in \mathbb{N}_{0}}^{\prime} l_{m} \log (m+\alpha) \neq 0 .
$$

Actually, if the latter inequality is not true, then

$$
\prod_{p \in \mathbb{P}}^{\prime} p^{k_{p}} \prod_{m \in \mathbb{N}_{0}}^{\prime}(m+\alpha)^{l_{m}}=1 .
$$

From this, it follows that

$$
\prod_{m \in \mathbb{N}_{0}}^{\prime}(m+\alpha)^{l_{m}}
$$

is a rational number. However, this contradicts the transcendence of $\alpha$. If all $l_{m}=0$, then $\sum_{p \in \mathbb{P}}^{\prime} k_{p} \log p \neq 0$ because the set $\{\log p: p \in \mathbb{P}\}$ is linearly independent over the field of rational numbers. Thus, (2.3) is true. Now, by (2.1), we find

$$
\begin{aligned}
g_{T, w}(\underline{k}, \underline{l}) & =\frac{1}{-i W(T, w) A(\underline{k}, \underline{l})} \int_{T_{0}}^{T} w(\tau) \mathrm{d} \exp \{-i \tau A(\underline{k}, \underline{l})\} \\
& \ll(W(T, w)|A(\underline{k}, \underline{l})|)^{-1}\left(1+\int_{T_{0}}^{T}|\mathrm{~d} w(\tau)|\right) \ll(W(T, w)|A(\underline{k}, \underline{l})|)^{-1}
\end{aligned}
$$

in view of a property of the variation of $w(\tau)$. Since $\lim _{T \rightarrow \infty} W(T, w)=\infty$, this shows that

$$
\lim _{T \rightarrow \infty} g_{T, w}(\underline{k}, \underline{l})=0 .
$$

Therefore, by (2.2),

$$
\lim _{T \rightarrow \infty} g_{T, w}(\underline{k}, \underline{l})= \begin{cases}1 & \text { if }(\underline{k}, \underline{l})=(\underline{0}, \underline{0}), \\ 0 & \text { if }(\underline{k}, \underline{l}) \neq(\underline{0}, \underline{0})\end{cases}
$$

and the theorem is proved because the right-hand side of the latter equality is the Fourier transform of the Haar measure $m_{H}$.

\section{Case of absolute convergence}

Theorem 3 implies a weighted joint limit theorem in the space $H^{2}(D)$, where $H(D)$ is the space of analytic functions on $D$ endowed with the topology of uniform convergence on compacta. Thus, let $\theta>1 / 2$ be a fixed number, for $m, n \in \mathbb{N}$,

$$
v_{n}(m)=\exp \left\{-\left(\frac{m}{n}\right)^{\theta}\right\}
$$

and, for $m \in \mathbb{N}_{0}, n \in \mathbb{N}$,

$$
v_{n}(m, \alpha)=\exp \left\{-\left(\frac{m+\alpha}{n+\alpha}\right)^{\theta}\right\} \text {. }
$$


Define the series

$$
\zeta_{n}(s)=\sum_{m=1}^{\infty} \frac{v_{n}(m)}{m^{s}} \quad \text { and } \quad \zeta_{n}(s, \alpha)=\sum_{m=0}^{\infty} \frac{v_{n}(m, \alpha)}{(m+\alpha)^{s}} .
$$

Then the latter series are absolutely convergent for $\sigma>1 / 2$, see $[13,23]$, respectively. For brevity, let

$$
\underline{\zeta}_{n}(s, \alpha)=\left(\zeta_{n}(s), \zeta_{n}(s, \alpha)\right) .
$$

Extend the functions $\omega_{1}(p)$, to the set $\mathbb{N}$ by the formula

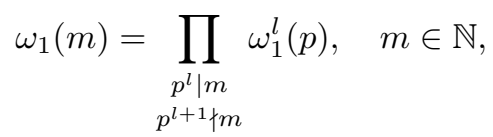

and, additionally to $\zeta_{n}(s)$ and $\zeta_{n}(s, \alpha)$, define

$$
\zeta_{n}\left(s, \omega_{1}\right)=\sum_{m=1}^{\infty} \frac{\omega_{1}(m) v_{n}(m)}{m^{s}} \quad \text { and } \quad \zeta_{n}\left(s, \omega_{2}, \alpha\right)=\sum_{m=0}^{\infty} \frac{\omega_{2}(m) v_{n}(m, \alpha)}{(m+\alpha)^{s}}
$$

and put $\underline{\zeta}_{n}(s, \omega, \alpha)=\left(\zeta_{n}\left(s, \omega_{1}\right), \zeta_{n}\left(s, \omega_{2}, \alpha\right)\right)$. Obviously, the series $\zeta_{n}\left(s, \omega_{1}\right)$ and $\zeta_{n}\left(s, \omega_{2}, \alpha\right)$ are absolutely convergent for $\sigma>1 / 2$ as well.

Consider the function $u_{n}: \Omega \rightarrow H^{2}(D)$ given by $u_{n}(\omega)=\underline{\zeta}_{n}(s, \omega, \alpha)$. Since the above seeries are absolutely convergent for $\sigma>1 / 2$, the function $u_{n}(\omega)$ is continuous. For $A \in \mathcal{B}\left(H^{2}(D)\right)$, define

$$
P_{T, n, w}(A)=\frac{1}{W(T, w)} \int_{T_{0}}^{T} w(\tau) I\left(\left\{\tau \in\left[T_{0}, T\right]: \underline{\zeta}_{n}(s+i \tau, \alpha) \in A\right\}\right) \mathrm{d} \tau .
$$

Then we have $P_{T, n, w}(A)=Q_{T, w}\left(u^{-1} A\right)$. Thus, the equality $P_{T, n, w}=Q_{T, w} u^{-1}$ is true. This, the continuity of $u_{n}$, Theorem 3 together with Theorem 5.1 of [4] lead to the following theorem.

Theorem 4. Suppose that $\alpha$ is transcendental and $w \in W$. Then $P_{T, n, w}$ converges weakly to the measure $V_{n} \stackrel{\text { def }}{=} m_{H} u_{n}^{-1}$ as $T \rightarrow \infty$.

The measure $V_{n}$ plays an important role in the proof of the limit theorem for

$$
\begin{array}{r}
P_{T, w}(A)=\frac{1}{W(T, w)} \int_{T_{0}}^{T} w(\tau) I\left(\left\{\tau \in\left[T_{0}, T\right]: \underline{\zeta}(s+i \tau, \alpha) \in A\right\}\right) \mathrm{d} \tau, \\
A \in \mathcal{B}\left(H^{2}(D)\right),
\end{array}
$$

where $\zeta(s, \alpha)=(\zeta(s), \zeta(s, \alpha))$. From the proof of the Mishou theorem [29], the following properties of $V_{n}$ follows. On the probability space $\left(\Omega, \mathcal{B}(\Omega), m_{H}\right)$, define the $H^{2}(D)$-valued random element

$$
\underline{\zeta}(s, \omega, \alpha)=\left(\prod_{p \in \mathbb{P}}\left(1-\frac{\omega_{1}(p)}{p^{s}}\right)^{-1}, \sum_{m=0}^{\infty} \frac{\omega_{2}(m)}{(m+\alpha)^{s}}\right),
$$


and let $P_{\underline{\zeta}}$ be the distribution of $\underline{\zeta}(s, \omega, \alpha)$, i. e.,

$$
P_{\underline{\zeta}}(A)=m_{H}\{\omega \in \Omega: \underline{\zeta}(s, \omega, \alpha) \in A\}, \quad A \in \mathcal{B}\left(H^{2}(D)\right) .
$$

Moreover, let $S=\{g \in H(D): g(s) \neq 0$ or $g(s) \equiv 0\}$. Under the above notation, we have

Lemma 1. Suppose that $\alpha$ is transcendental. Then $V_{n}$ converges weakly to $P_{\underline{\zeta}}$ as $n \rightarrow \infty$. Moreover, the support of $P_{\underline{\zeta}}$ is the set $S \times H(D)$.

To prove that $P_{T, w}$, as $T \rightarrow \infty$, also converges weakly to the measure $P_{\underline{\zeta}}$, some approximation of $\underline{\zeta}(s, \alpha)$ by $\underline{\zeta}_{n}(s, \alpha)$ is needed.

\section{Approximation in the mean}

For $g_{1}, g_{2} \in H(D)$, define

$$
\rho\left(g_{1}, g_{2}\right)=\sum_{l=1}^{\infty} 2^{-l} \frac{\sup _{s \in K_{l}}\left|g_{1}(s)-g_{2}(s)\right|}{1+\sup _{s \in K_{l}}\left|g_{1}(s)-g_{2}(s)\right|},
$$

where $\left\{K_{l}: l \in \mathbb{N}\right\} \subset D$ is a sequence of compact subsets such that $D=\bigcup_{l=1}^{\infty} K_{l}$, $K_{l} \subset K_{l+1}$ for all $l \in \mathbb{N}$, and if $K \subset D$ is a compact set, then $K$ lies in some $K_{l}$. Then $\rho$ is a metric on $H(D)$ that induces its topology of uniform convergence on compacta.

Now, let $\underline{g}_{1}=\left(g_{11}, g_{12}\right), \underline{g}_{2}=\left(g_{21}, g_{22}\right) \in H^{2}(D)$. Then putting

$$
\underline{\rho}\left(\underline{g}_{1}, \underline{g}_{2}\right)=\max _{1 \leqslant j \leqslant 2} \rho\left(g_{1 j}, g_{2 j}\right)
$$

gives a metric on $H^{2}(D)$ inducing the product topology.

The following statement is true.

Theorem 5. Suppose that $w \in W$. Then

$$
\lim _{n \rightarrow \infty} \limsup _{T \rightarrow \infty} \frac{1}{W(T, w)} \int_{T_{0}}^{T} w(\tau) \underline{\rho}\left(\underline{\zeta}(s+i \tau, \alpha), \underline{\zeta}_{n}(s+i \tau, \alpha)\right) \mathrm{d} \tau=0
$$

for all $0<\alpha \leqslant 1$.

Proof. By the definition of the metric $\rho$, it suffices to prove the equalities

$$
\lim _{n \rightarrow \infty} \limsup _{T \rightarrow \infty} \frac{1}{W(T, w)} \int_{T_{0}}^{T} w(\tau) \rho\left(\zeta(s+i \tau), \zeta_{n}(s+i \tau)\right) \mathrm{d} \tau=0
$$

and

$$
\lim _{n \rightarrow \infty} \limsup _{T \rightarrow \infty} \frac{1}{W(T, w)} \int_{T_{0}}^{T} w(\tau) \rho\left(\zeta(s+i \tau, \alpha), \zeta_{n}(s+i \tau, \alpha)\right) \mathrm{d} \tau=0 .
$$


Obviously, (4.1) is a corollary of (4.2) with $\alpha=1$. Moreover, to prove (4.2) it suffices to show that, for every compact set $K \subset D$,

$$
\lim _{n \rightarrow \infty} \limsup _{T \rightarrow \infty} \frac{1}{W(T, w)} \int_{T_{0}}^{T} w(\tau) \sup _{s \in K}\left|\zeta(s+i \tau, \alpha)-\zeta_{n}(s+i \tau, \alpha)\right| \mathrm{d} \tau=0 .
$$

Let

$$
l_{n}(s, \alpha)=\frac{s}{\theta} \Gamma\left(\frac{s}{\theta}\right)(n+\alpha)^{s}, \quad n \in \mathbb{N},
$$

where $\Gamma(s)$ is the Euler gamma-function. Then the classical Mellin formula implies, for $\sigma>1 / 2$, the equality

$$
\zeta_{n}(s, \alpha)=\frac{1}{2 \pi i} \int_{\theta-i \infty}^{\theta+i \infty} \zeta(s+z, \alpha) l_{n}(z, \alpha) \frac{\mathrm{d} z}{z} .
$$

We take an arbitrary compact set $K \subset D$, and fix $\varepsilon>0$ such that $1 / 2+2 \varepsilon \leqslant$ $\sigma \leqslant 1-\varepsilon$ for points $s=\sigma+i v \in K$. Then, by (4.4) and the residue theorem, for $\theta_{1}>0$,

$$
\zeta_{n}(s, \alpha)-\zeta(s, \alpha)=\frac{1}{2 \pi i} \int_{-\theta_{1}-i \infty}^{-\theta_{1}+i \infty} \zeta(s+z, \alpha) l_{n}(z, \alpha) \frac{\mathrm{d} z}{z}+R_{n}(s, \alpha),
$$

where $R_{n}(s, \alpha)=l_{n}(1-s, \alpha) /(1-s)$. Suppose that $\theta_{1}=\sigma-\varepsilon-1 / 2$. Then (4.5) shows that, for $s \in K$,

$$
\begin{aligned}
\left|\zeta_{n}(s, \alpha)-\zeta(s, \alpha)\right| \leqslant & \frac{1}{2 \pi} \int_{-\infty}^{\infty}\left|\zeta\left(s+i \tau-\theta_{1}+i t, \alpha\right)\right| \frac{\left|l_{n}\left(-\theta_{1}+i t, \alpha\right)\right|}{\left|-\theta_{1}+i t\right|} \mathrm{d} t \\
& +\left|R_{n}(s+i \tau, \alpha)\right| .
\end{aligned}
$$

Hence, after shifting $v+t$ to $t$, we obtain

$$
\frac{1}{W(T, w)} \int_{T_{0}}^{T} w(\tau) \sup _{s \in K}\left|\zeta(s+i \tau, \alpha)-\zeta_{n}(s+i \tau, \alpha)\right| \mathrm{d} \tau \ll I_{1}+I_{2},
$$

where

$$
\begin{aligned}
I_{1}= & \int_{-\infty}^{\infty} w(\tau)\left(\frac{1}{W(T, w)} \int_{T_{0}}^{T}|\zeta(1 / 2+\varepsilon+i(t+\tau), \alpha)| \mathrm{d} \tau\right) \\
& \times \sup _{s \in K} \frac{\left|l_{n}(1 / 2+\varepsilon-s+i t, \alpha)\right|}{|1 / 2+\varepsilon-s+i t|} \mathrm{d} t, \\
I_{2}= & \frac{1}{W(T, w)} \int_{T_{0}}^{T} w(\tau) \sup _{s \in K}\left|R_{n}(s+i \tau, \alpha)\right| \mathrm{d} \tau .
\end{aligned}
$$

It is well known that $\Gamma(\sigma+i t) \ll \exp \{-c|t|\}$ uniformly in $\sigma_{1} \leqslant \sigma \leqslant \sigma_{2}$ for every $\sigma_{1}<\sigma_{2}$ with an absolute constant $c>0$. Therefore, putting $\theta=1 / 2+\varepsilon$, we find that, for $s \in K$,

$$
\begin{aligned}
& \frac{\left|l_{n}(1 / 2+\varepsilon-s+i t, \alpha)\right|}{|1 / 2+\varepsilon-s+i t|}=\frac{(n+\alpha)^{1 / 2+\varepsilon-\sigma}}{\theta}\left|\Gamma\left(\frac{1 / 2+\varepsilon-\sigma}{\theta}+\frac{i(t-v)}{\theta}\right)\right| \\
& \ll_{\alpha} \frac{n^{-\varepsilon}}{\theta} \exp \left\{-c \frac{|t-v|}{\theta}\right\} \ll_{K, \alpha} n^{-\varepsilon} \exp \left\{-c_{1}|t|\right\}
\end{aligned}
$$


with $c_{1}>0$. In [3] it was obtained that, for $\sigma, 1 / 2<\sigma<1$, and $t \in \mathbb{R}$,

$$
\int_{T_{0}}^{T} w(\tau)|\zeta(\sigma+i(t+\tau), \alpha)|^{2} \mathrm{~d} t \ll W(t, w)\left(1+|t|^{2}\right) .
$$

Hence,

$$
\begin{aligned}
& \int_{T_{0}}^{T} w(\tau)|\zeta(\sigma+i(t+\tau), \alpha)|^{2} \mathrm{~d} \tau \\
& \ll\left(\int_{T_{0}}^{T} w(\tau) \mathrm{d} \tau \int_{T_{0}}^{T} w(\tau)|\zeta(1 / 2+\varepsilon+i(t+\tau), \alpha)|^{2} \mathrm{~d} \tau\right)^{1 / 2} \ll W(t, w)\left(1+|t|^{2}\right) .
\end{aligned}
$$

This together with (4.7) shows that

$$
I_{1} \ll_{K} n^{-\varepsilon} \int_{-\infty}^{\infty}(1+|t|) \exp \left\{-c_{1}|t|\right\} \mathrm{d} t \ll_{K, \alpha} n^{-\varepsilon} .
$$

Similarly, we find that, for $s \in K$,

$$
\left|R_{n}(s+i \tau, \alpha)\right| \ll_{\alpha} n^{1-\sigma} \exp \left\{-c \frac{|\tau-v|}{\theta}\right\} \ll_{K, \alpha} n^{1-\sigma} \exp \left\{-c_{2}|\tau|\right\}
$$

with $c_{2}>0$. Thus,

$$
I_{2} \ll_{K, \alpha} n^{1 / 2-2 \varepsilon} \frac{1}{W(T, w)} \int_{T_{0}}^{T} w(\tau) \exp \left\{-c_{2}|\tau|\right\} \mathrm{d} \tau \ll_{K, \alpha} \frac{n^{1 / 2-2 \varepsilon}}{W(T, w)} .
$$

If $T \rightarrow \infty$, then $I_{2} \rightarrow 0$, because $W(T, w) \rightarrow \infty$. Moreover, by (4.8), if $n \rightarrow \infty$, then $I_{1} \rightarrow 0$. Therefore, (4.6) implies (4.3). The lemma is proved.

\section{A limit theorem for $\underline{\zeta}(s, \alpha)$}

Now we are ready to prove the weak convergence for $P_{T, w}$ as $T \rightarrow \infty$.

Theorem 6. Suppose that $\alpha$ is transcendental and $w \in W$. Then $P_{T, w}$ converges weakly to the measure $P_{\underline{\zeta}}$ as $T \rightarrow \infty$.

Proof. On a certain probability space with measure $\mu$, define a random variable $\theta_{T, w}$ by

$$
\mu\left\{\theta_{T, w} \in A\right\}=\frac{1}{W(T, w)} \int_{T_{0}}^{T} w(\tau) I(A) \mathrm{d} \tau, \quad A \in \mathcal{B}(\mathbb{R}) .
$$

Consider the $H^{2}(D)$-valued random element

$$
\underline{X}_{T, n, w}=\underline{X}_{T, n, w}(s)=\underline{\zeta}_{n}\left(s+i \theta_{T, w}, \alpha\right) .
$$

Then, in view of Theorem 4,

$$
\underline{X}_{T, n, w} \underset{T \rightarrow \infty}{\stackrel{\mathcal{D}}{\longrightarrow}} Y_{n}
$$


where $Y_{n}$ is the $H^{2}(D)$-valued random element with the distribution $V_{n}$. Lemma 1 implies the relation

$$
Y_{n} \underset{n \rightarrow \infty}{\stackrel{\mathcal{D}}{\longrightarrow}} P_{\underline{\zeta}} .
$$

Moreover, an application of Theorem 5 shows that, for every $\varepsilon>0$,

$$
\begin{aligned}
& \lim _{n \rightarrow \infty} \limsup _{T \rightarrow \infty} \mu\left(\underline{\rho}\left(\underline{X}_{T, w}(s), \underline{X}_{T, n, w}(s)\right) \geqslant \varepsilon\right) \\
& \quad \ll \lim _{n \rightarrow \infty} \limsup _{T \rightarrow \infty} \frac{1}{\varepsilon W(T, w)} \int_{T_{0}}^{T} w(\tau) \underline{\rho}\left(\underline{\zeta}(s+i \tau, \alpha), \underline{\zeta}_{n}(s+i \tau, \alpha)\right) \mathrm{d} \tau=0,
\end{aligned}
$$

where the $H^{2}(D)$-valued random element $\underline{X}_{T, w}=\underline{X}_{T, w}(s)$ is defined by

$$
\underline{X}_{T, w}(s)=\underline{\zeta}\left(s+i \theta_{T, w}, \alpha\right) .
$$

Now, relations (5.1)-(5.2) show that all hypotheses of Theorem 4.2 from [4] are satisfied. Therefore, we obtain that

$$
\underline{X}_{T, w} \underset{T \rightarrow \infty}{\stackrel{\mathcal{D}}{\longrightarrow}} P_{\underline{\zeta}},
$$

and this is equivalent to the assertion of the theorem.

\section{Proof of universality}

Theorem 2 follows easily from Theorem 6 and the Mergelyan theorem on the approximation of analytic functions by polynomials [28].

Proof. (Proof of Theorem 2). By the Mergelyan theorem, there exist polynomials $p_{1}(s)$ and $p_{2}(s)$ such that

$$
\sup _{s \in K_{1}}\left|f_{1}(s)-\mathrm{e}^{p_{1}(s)}\right|<\frac{\varepsilon}{2}, \quad \sup _{s \in K_{2}}\left|f_{2}(s)-p_{2}(s)\right|<\frac{\varepsilon}{2} .
$$

Define the set

$$
G_{\varepsilon}=\left\{g_{1}, g_{2} \in H(D): \sup _{s \in K_{1}}\left|g_{1}(s)-\mathrm{e}^{p_{1}(s)}\right|<\frac{\varepsilon}{2}, \sup _{s \in K_{2}}\left|g_{2}(s)-p_{2}(s)\right|<\frac{\varepsilon}{2}\right\} .
$$

We observe that, in virtue of Lemma $1,\left(\mathrm{e}^{p_{1}(s)}, p_{2}(s)\right)$ is an element of the support of the measure $P_{\zeta}$. Since $G_{\varepsilon}$ is an open neighbourhood of an element of the support of $P_{\underline{\xi}}$, the inequality

$$
P_{\underline{\zeta}}\left(G_{\varepsilon}\right)>0
$$

is true. Therefore, using the equivalent of the weak convergence of probability measures in terms of open sets and taking into account Theorem 6 , we have

$$
\liminf _{T \rightarrow \infty} P_{T, w}\left(G_{\varepsilon}\right) \geqslant P_{\underline{\underline{\zeta}}}\left(G_{\varepsilon}\right)>0 .
$$


Hence, by the definitions of $P_{T, w}$ and $G_{\varepsilon}$,

$$
\begin{gathered}
\liminf _{T \rightarrow \infty} \frac{1}{W(T, w)} \int_{T_{0}}^{T} w(\tau) I\left(\left\{\tau \in\left[T_{0}, T\right]: \sup _{s \in K_{1}}\left|\zeta(s+i \tau)-\mathrm{e}^{p_{1}(s)}\right|<\frac{\varepsilon}{2}\right.\right. \\
\left.\left.\sup _{s \in K_{2}}\left|\zeta(s+i \tau, \alpha)-p_{2}(s)\right|<\frac{\varepsilon}{2}\right\}\right) \mathrm{d} \tau>0 .
\end{gathered}
$$

It remains to replace $\mathrm{e}^{p_{1}(s)}$ and $p_{2}(s)$ by $f_{1}(s)$ and $f_{2}(s)$, respectively. Suppose that $\tau$ satisfy inequalities

$$
\sup _{s \in K_{1}}\left|\zeta(s+i \tau)-\mathrm{e}^{p_{1}(s)}\right|<\frac{\varepsilon}{2}, \quad \sup _{s \in K_{2}}\left|\zeta(s+i \tau, \alpha)-p_{2}(s)\right|<\frac{\varepsilon}{2} .
$$

Then inequalities (6.1) imply

$$
\sup _{s \in K_{1}}\left|\zeta(s+i \tau)-f_{1}(s)\right|<\varepsilon, \quad \sup _{s \in K_{2}}\left|\zeta(s+i \tau, \alpha)-f_{2}(s)\right|<\varepsilon .
$$

Consequently,

$$
\begin{aligned}
& \left\{\tau \in\left[T_{0}, T\right]: \sup _{s \in K_{1}}\left|\zeta(s+i \tau)-\mathrm{e}^{p_{1}(s)}\right|<\frac{\varepsilon}{2}, \sup _{s \in K_{2}}\left|\zeta(s+i \tau, \alpha)-p_{2}(s)\right|<\frac{\varepsilon}{2}\right\} \\
& \quad \subset\left\{\tau \in\left[T_{0}, T\right]: \sup _{s \in K_{1}}\left|\zeta(s+i \tau)-f_{1}(s)\right|<\varepsilon, \sup _{s \in K_{2}}\left|\zeta(s+i \tau, \alpha)-f_{2}(s)\right|<\varepsilon\right\} .
\end{aligned}
$$

This and (6.3) prove the first assertion of the theorem.

Define one more set

$$
\hat{G}_{\varepsilon}=\left\{g_{1}, g_{2} \in H(D): \sup _{s \in K_{1}}\left|g_{1}(s)-f_{1}(s)\right|<\varepsilon, \sup _{s \in K_{2}}\left|g_{2}(s)-f_{2}(s)\right|<\varepsilon\right\} .
$$

Then the boundaries $\partial \hat{G}_{\varepsilon_{1}}$ and $\partial \hat{G}_{\varepsilon_{2}}$ do not intersect for different positive $\varepsilon_{1}$ and $\varepsilon_{2}$. This shows that the set $\hat{G}_{\varepsilon}$ is a continuity set of the measure $P_{\zeta}$ for all but at most countably many $\varepsilon>0$. Therefore, using the equivalent of weak convergence of probability measures in terms of continuity sets, we obtain by Theorem 6 that

$$
\lim _{T \rightarrow \infty} P_{T, w}\left(\hat{G}_{\varepsilon}\right)=P_{\underline{\zeta}}\left(\hat{G}_{\varepsilon}\right)
$$

for all but at most countably many $\varepsilon>0$. Moreover, inequalities (6.1) imply the inclusion $G_{\varepsilon} \subset \hat{G}_{\varepsilon}$. Thus, by (6.2), the inequality $P_{\underline{\zeta}}\left(\hat{G}_{\varepsilon}\right)>0$ holds. This, the definitions of $P_{T, w}$ and $\hat{G}_{\varepsilon}$, and (6.4) prove the second assertion of the theorem.

\section{Acknowledgements}

The research of the first author is funded by the European Social Fund (project No. 09.3.3-LMT-K-712-01-0037) under grant agreement with the Research Council of Lithuania (LMT LT). 


\section{References}

[1] B. Bagchi. The Statistical Behaviuor and Universality Properties of the Riemann Zeta-Function and Other Allied Dirichlet Series. PhD Thesis, Indian Statistical Institute, Calcutta, 1981.

[2] A. Balčiūnas, V. Garbaliauskienè, J. Karaliūnaitè, R. Macaitienè, J. Petuškinaitè and A. Rimkevičienè. Joint discrete approximation of a pair of analytic functions by periodic zeta-functions. Math. Modell. Anal., 25(1):71-87, 2020. https://doi.org/10.3846/mma.2020.10450.

[3] A. Balčiūnas and G. Vadeikis. A weighted universality throrem for the Hurwitz zeta-function. Šiauliai Math. Semin., 12(20):5-18, 2017.

[4] P. Billingsley. Convergence of Probability Measures. Willey, New York, 1968.

[5] V. Garbaliauskienè, J. Karaliūnaitè and A. Laurinčikas. On zeros of some combinations of Dirichlet $L$-functions and Hurwitz zeta-functions. Math. Modell. Anal., 22(6):733-749, 2017. https://doi.org/10.3846/13926292.2017.1365313.

[6] S.M. Gonek. Analytic Properties of Zeta and L-Functions. PhD Thesis, University of Michigan, 1979.

[7] R. Kačinskaitè and B. Kazlauskaitè. Two remarks related to the universality of zeta-functions with periodic coefficients. Results Math., 73(3):95, 2018. https://doi.org/10.1007/s00025-018-0856-z.

[8] R. Kačinskaitè and A. Laurinčikas. The joint distribution of periodic zeta-functions. Studia Sci. Math. Hungarica, 48(2):257-279, 2011. https://doi.org/10.1556/sscmath.48.2011.2.1162.

[9] R. Kačinskaitè and K. Matsumoto. The mixed joint universality for a class of zeta-functions. Math. Nachr., 288(16):1900-1909, 2015. https://doi.org/10.1002/mana.201400366.

[10] R. Kačinskaitè and K. Matsumoto. Remarks on the mixed joint universality for a class of zeta-functions. Bull. Austral. Math. Soc., 95(2):187-198, 2017. https://doi.org/10.1017/S0004972716000733.

[11] R. Kačinskaitè and K. Matsumoto. On mixed joint discrete universality for a class of zeta-functions. II. Lith. Math. J., 59(1):54-66, 2019. https://doi.org/10.1007/s10986-019-09432-1.

[12] A. Laurinčikas. On the universality of the Riemann zeta-function. Lith. Math. J., 35(4):399-402, 1995. https://doi.org/10.1007/BF02348827.

[13] A. Laurinčikas. Limit Theorems for the Riemann Zeta-Function. Kluwer Academic Publishers, Dordrecht, Boston, London, 1996. https://doi.org/10.1007/978-94-017-2091-5.

[14] A. Laurinčikas. The joint universality of Hurwitz zeta-functions. Šiauliai Math. Semin., 3(11):169-187, 2008.

[15] A. Laurinčikas. Joint universality of zeta-functions with periodic coefficients. Izv. Math., $\mathbf{7 4}(3): 515-539, \quad 2010$. https://doi.org/10.1070/IM2010v074n03ABEH002497.

[16] A. Laurinčikas. Universality theorems for zeta-functions with periodic coefficients. Siber. Math. J., $\quad \mathbf{5 7}(2): 330-339,2016$. https://doi.org/10.1134/S0037446616020154.

[17] A. Laurinčikas. A discrete version of the Mishou theorem. II. Proc. Steklov Inst. Math., 296(1):172-182, 2017. https://doi.org/10.1134/S008154381701014X. 
[18] A. Laurinčikas. Joint value distribution theorems for the Riemann and Hurwitz zeta-functions. Moscow Math. J., 18(2):349-366, 2018. https://doi.org/10.17323/1609-4514-2018-18-2-349-366.

[19] A. Laurinčikas. Joint discrete universality for periodic zeta-functions. Quaest. Math., 42(5):687-699, 2019. https://doi.org/10.2989/16073606.2018.1481891.

[20] A. Laurinčikas. Non-trivial zeros of the Riemann zeta-function and joint universality theorems. J. Math. Anal. Appl., 475(1):385-402, 2019. https://doi.org/10.1016/j.jmaa.2019.02.047.

[21] A. Laurinčikas. On the Mishou theorem with algebraic parameter. Siber. Math. J., 60(6):1075-1082, 2019. https://doi.org/10.1134/S0037446619060144.

[22] A. Laurinčikas. Joint discrete universality for periodic zeta-functions. II. Quaest. Math., 2020. https://doi.org/10.2989/16073606.2019.1654554.

[23] A. Laurinčikas and R. Garunkštis. The Lerch Zeta-Function. Kluwer Academic Publishers, Dordrecht, Boston, London, 2002. https://doi.org/10.1007/978-94017-6401-8.

[24] A. Laurinčikas and R. Macaitienè. Joint approximation of analytic functions by shifts of the Riemann and periodic Hurwitz zeta-functions. Appl. Anal. Discrete Math., 12(2):508-527, 2018. https://doi.org/10.2298/AADM170713016L.

[25] A. Laurinčikas, D. Šiaučiūnas and G. Vadeikis. Weighted discrete universality of the Riemann zeta-function. Math. Modell. Anal., 25(1):21-36, 2020. https://doi.org/10.3846/mma.2020.10436.

[26] R. Macaitienè, M. Stoncelis and D. Šiaučiūnas. A weighted discrete universality theorem for periodic zeta-functions. II. Math. Modell. Analysis, 22(6):750-762, 2017. https://doi.org/10.3846/13926292.2017.1365779.

[27] R. Macaitienè, M. Stoncelis and D. Šiaučiūnas. A weighted universality theorem for periodic zeta-functions. Math. Modell. Analysis, 22(1):95-105, 2017. https://doi.org/10.3846/13926292.2017.1269373.

[28] S.N. Mergelyan. Uniform approximations to functions of complex variable. Usp. Mat. Nauk., 7:31-122, 1952 (in Russian).

[29] H. Mishou. The joint value distribution of the Rieman zeta-function and Hurwitz zeta-functions. Lith. Math. J., 47(1):32-47, 2007. https://doi.org/10.1007/s10986-007-0003-0.

[30] J. Steuding. Value-Distribution of L-Functions. Lecture Notes Math. vol. 1877, Springer, Berlin, Heidelberg, 2007. https://doi.org/10.5565/PUBLMAT_PJTN05_12.

[31] S.M. Voronin. Theorem on the "universality" of the Riemann zeta-function. Izv. Akad. Nauk SSSR, Ser. Matem., 39:475-486, 1975 (in Russian).

[32] S.M. Voronin. Analytic Properties of Generating Function of Arithmetic Objects. Diss. doktor fiz.-matem. nauk, Steklov Math. Inst., Moscow, 1977 (in Russian). 